The University of Akron

\title{
IdeaExchange@UAkron
}

Proceedings from the Document Academy

University of Akron Press Managed

December 2017

\section{Promoting Trustworthiness and Discoverability in an S-town of OSN Documents}

Laurie J. Bonnici

University of Alabama, lbonnici@ua.edu

Please take a moment to share how this work helps you through this survey. Your feedback will be important as we plan further development of our repository.

Follow this and additional works at: https://ideaexchange.uakron.edu/docam

Part of the Communication Technology and New Media Commons, Health Communication $\underline{\text { Commons, Library and Information Science Commons, and the Social Media Commons }}$

\section{Recommended Citation}

Bonnici, Laurie J. (2017) "Promoting Trustworthiness and Discoverability in an S-town of OSN Documents,"

Proceedings from the Document Academy: Vol. 4 : Iss. 2 , Article 5.

DOI: https://doi.org/10.35492/docam/4/2/5

Available at: https://ideaexchange.uakron.edu/docam/vol4/iss2/5

This Conference Proceeding is brought to you for free and open access by University of Akron Press Managed at IdeaExchange@UAkron, the institutional repository of The University of Akron in Akron, Ohio, USA. It has been accepted for inclusion in Proceedings from the Document Academy by an authorized administrator of

IdeaExchange@UAkron.For more information, please contact mjon@uakron.edu, uapress@uakron.edu. 


\section{Introduction}

For over a little more than a decade, online social networks (OSN) have offered a convenient channel for casual communication as well as information sharing. Facebook (FB) has evolved from a networking tool where young adults and particularly college students connect socially into a virtual forum where adults seek advice from others particularly when an authoritative information resource is lacking. Specialized groups on FB serve as an important information vector for cognitive authority presented through accounting of personal experience. These stories inform people in health information seeking crisis. These groups constitute an info-communicational resource that promotes circulation of secondhand knowledge to supplement a dearth of authority resources on the topic at hand. Furthermore, such groups provide social support to aid the psychosocial stress that frequently accompanies the initial stages of illness diagnosis. Part of a larger project on grounded theory building to identify and promote cognitive authority in OSN, this work in progress seeks to identify textual and visual markers that present as witness marks of quality secondhand knowledge. I propose witness marks as a framework to consider structuring folksonomies and codified norms for FB groups to increase the ability to retrieve and identify trustworthy information in OSN, specifically FB groups. First I discuss current social information retrieval (SIR) approaches resulting in a point of failure for searching the plethora of social interactions that constitute the fabric of documents that ensconce cognitive authority. Second I present the notion of witness marks and raise the question of their application in the OSN context to ameliorate discovery of second and knowledge. The purpose of this paper is to demonstrate how interaction design (IxD) can be applied to FB groups to both builds towards, and be reflective of, cognitive authority presentation and discoverability of secondhand knowledge.

\section{Social Information Retrieval (SIR)}

OSN communities are highly populous and manifest in a variation of understanding by the users of the system. This compounded by exponential connections within the social web present a vast amount of communication and information that is promoted, stored, and available to harvest in Facebook. This fabric of social documents presents an s-town ${ }^{1}$ of unwieldy information that eludes current SIR strategies.

The seemingly infinite amount of communication and information presented as documents offering content adorned with cognitive authority,

1 Originally coined in a podcast by Serial and This American Life https://www.wired.com/2017/03/s-town-podcast/ . In this instance it may refer to shit-town, social-town, search-town... 
intended to inform individuals in a health information seeking activity, was constructed well before the application of hashtags (\#) in FB. A mechanism for cobbling together posts on the same topic, the hashtag was not available in FB until 2013 leaving over a decade's worth of second hand knowledge unsearchable.

A recent study of two OSN groups, both with a stated purpose centered upon health information provision, revealed that group members rarely if ever employ the hashtag in community posts (Bonnici and Ma, in-press). The majority of posts revealed personal experiences in the form of practical advice for helping members in crisis make informed decisions. However, information seekers would need a high level of information literacy to sort out the credible material from unreliable sources in these two forums.

Further complicating the information-seeking puzzle is that current social information retrieval (SIR) classifications elude the FB group community structure (Bonnici and Ma, in-press). The complexity of FB eludes labeling by any one of three SIR classifications that address issues of symmetry and k-partite relationships. FB groups involve more than 1:1 relationships, rather they embody 1:many and many:1 relationships. For example, when a new member joins a specialized FB group they are frequently in information crisis due to the fact that authoritative resources are inaccessible (i.e. medical journals that are often unaffordable to lay populations) or the topic is highly politicized and stigmatized by power groups (e.g. vaccine injury). Sans access to authoritative information resource, the new community member posts an inquiry to the FB group community revealing a 1:many relationship. The inquiry fuels a community response revealing a collective knowledge shared by multiple community members intended to fill the information void revealing a many: 1 relationship. Throughout the question/response negotiation process between the new member and the community where the information need is teased out, micro-discussions may appear as personalized discussions often displaying social support such as well-wishes for the health condition (1:1 relationship). Current SIR mechanisms do not address systems that embody simultaneous symmetry/asymmetry conditions. Further complicating the SIR approach, the FB system does not allow inter-personal connectivity within posts, which is a signature strategy in k-partite systems. In essence, another member within the FB group cannot tag a message posted by another member. A prominent OSN example housed in a k-partite system is Goodreads. Users in the Goodreads community can tag the posts of other users to add relevancy and inter-personal messages. The failure of FB groups to be classified exclusively into a symmetric, asymmetric, or k-partite relationship eludes current SIR strategies. This condition leaves a wealth of secondhand knowledge systematically unsearchable. 


\section{Witness Marks}

How do we commence transforming an s-town of socially constructed documents into a searchable community of secondhand knowledge? Effused by the advent of computer systems, information scientists and computer scientists seek to improve their retrieval systems by examining the interactions between humans and computers. Known as human-computer-interaction (HCI), researchers observe the ways in which humans interact with computers and in turn inform system design that let humans interact with computers more efficiently (Card, Moran, \& Newell, 1980). However, in this presentation I am not observing human use. For based on the above analysis of SIR strategies, any human attempt to search FB results in inconsistencies and failures. Thus, I embark upon a path of 'what could be' in the interest of satisfying the majority of users who turn to specialized FB groups to inform important decisions in their lives.

Interaction design (IxD) approaches seek to synthesize and imagine things as they could be, distinguishing it as design rather than a scientific approach (Cooper, Reiman, \& Cronin, 2007). Yet it is often this open thought approach to 'how' could users search that informs the 'what' could be in the functional sense of search in FB. IxD focuses on behaviors leading to the "design of interactive products to support the way people communicate and interact in their everyday and working lives" (Rogers, Sharp, \& Preece, 2011). The design approach allows for creative thinking about how FB documents could be efficiently and effectively searchable and believable or trustworthy. The beauty in applying an IxD lens to the FB search conundrum is that the design approach has also been employed when creating non-digital products, exploring how a user might interact with physical entities. Stepping willfully off of the beaten path, I ponder the notion of witness marks as a way of thinking about what could provide a human-assisted strategy to aid users in locating and whittling out the drivel from an s-town of FB documents.

Clocks present as physical objects that, up to the advent of the digital clock, were mechanically constructed. Their endurance meant that they outlived or traveled beyond the reaches of their creator. Clocks constructed of wooden gears and rope pulleys built by hand over 200 years ago adorn the walls of modern homes and find their way into clock shops today for repair. Sans an instruction manual, these antique devices require reverse engineering strategies to discern functioning systems as a strategy to diagnose the missing beat of the nonfunctioning timepiece.

Traditionally employed in horology, clockmakers left witness marks intentionally or accidentally as indicators of certain facts in clock functioning. Witness marks left in the inner workings over the clock's lifetime encouraged understanding of what once was (Maltese, 2017). The ensuing image conveys the witness mark in the Pouvillion Restoration Project. The witness marks in the 
original Easter calculator system provide information in how the calculator system was made. Despite being pointed out, the witness mark is difficult to see in Figure 1. Yet is strategically informing to the clock repairman who knows and understands witness marks as part of professional knowledge.

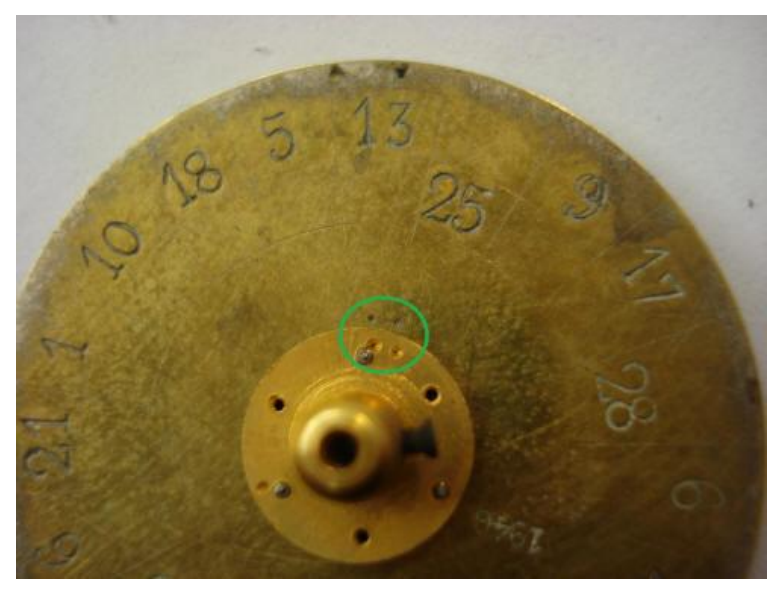

Figure 1: Dial carrying the calculated Easter dates (Source: My-time-machines.net)

Beyond horology, in an effort to aid professionals in their work, witness marks have been adopted and adapted by manufacturing and forensics. Each discipline offers unique perspectives in how witness marks can be uniquely deployed to accommodate the intentions of specific situations.

In forensics, unlike horology, criminals and their victims unintentionally leave witness marks. Often referred to as trace evidence, these witness marks aid criminal investigators in reconstructing the crime. For example, bite marks on a victim are photographed using high-quality imagery and can be matched to a suspect's teeth. Figure 2 shows teeth marks matched to teeth as evidence in a sexual assault case. 


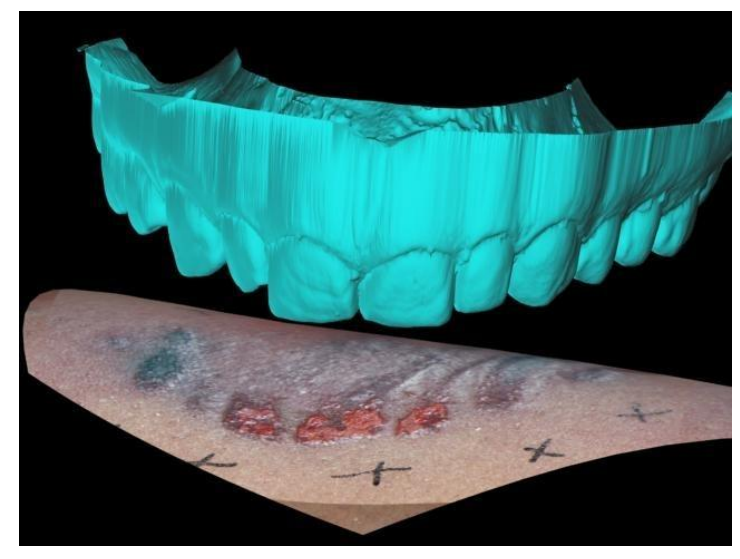

Figure 2: Reconstruction of bitemark (Source: Visible Proofs)

In the manufacturing context, witness marks or witness lines are intentionally casted to indicate alignment for proper assembly of parts. These visual references allow individuals other than the designers and machinists to disassemble, repair, and reassemble major machinery. Figure 3 is an example of a witness mark cast in the tool and die process indicating where the two discs align for proper assembly.

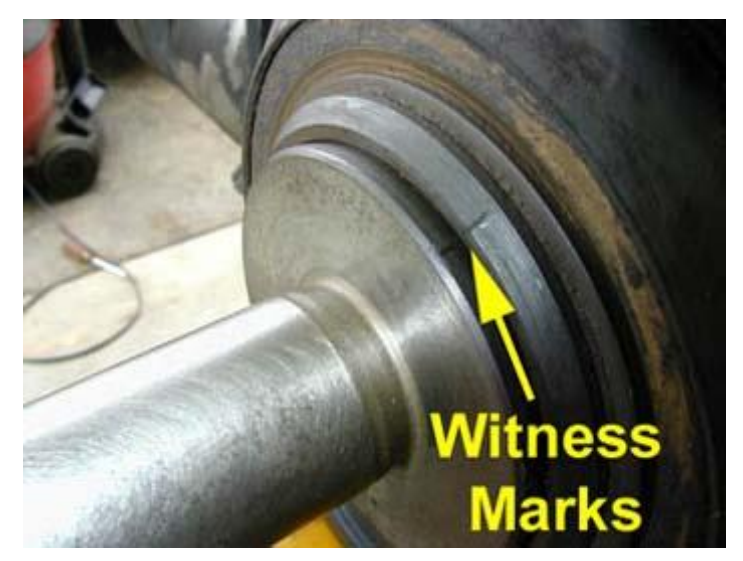

Figure 3: Lathe chuck alignment (Source: Softsoldier.com)

\section{Interactive Design for Social Media}

Well established in other disciplines, what of the application of witness marks for locating and identifying quality secondhand knowledge in OSN? Here I ponder their application for information science. Like the examples above in horology, forensics, and manufacturing, a user could leave witness marks in social media 
posts wittingly or unwittingly. When considering IxD, intentionally applied approaches serve to build a system that supports discoverability and promotes trustworthy information. Prior to discussing currently available strategies in the FB system that render as witness marks, I offer an example of several unwitting occurrences I have noted in examinations of FB groups. I offer examples from a FB public group centered upon a canine chronic illness to support the notion of witness marks for OSN. In this group the majority of originating posts are from owners whose dog was recently diagnosed with the chronic condition that is the topic this group addresses. For instance, "my dog was diagnosed with XX 2 weeks ago..." The short period of time ( 2 weeks) unwittingly indicates a newbie to the disease and thus limited knowledge on the topic. Any recommendations or advice offered within the post would raise question as to the trustworthiness of cognitive authority of the group member. This example introduces the notion of time as a witness mark when considering cognitive authority presentation. Although unwittingly offered in this instance, time expressed intentionally could build presentation of cognitive authority. Consider the following post as it relates to time. "My rottie has been taking XXX [medication name] for a little over 10 years. Her appetite returned after a few weeks." Given the length of time the dog has been on medication, we could assume that the owner has established cognitive authority having dealt with the disease for more than a decade.

When considering factors of time in the canine chronic disease FB group, employing a small icon to the user profile within the FB group area would convey years of experience or length of time a user has been a member of the group. The icon as witness mark aids the information-seeking user in determining quality and trustworthiness of second-hand knowledge. Time, among other factors, could be considered in search algorithms when ranking results. The higher the number of years of experience a user has, the higher the ranking in the search results.

Another factor contributing to information discovery and cognitive authority identification is the consideration of 'who' is delivering the information. Again, this can either be an intentionally provided augmentation to user identification or unwitting. For example in the canine chronic illness forum, a post that contains "vet here" labels the community member as expert rather than a source of cognitive authority (lay experience). "Vet here" is a witness mark intentionally left by the poster. An example of unwitting identification is inherent to group administrator status. If a user seeking information recognizes a discussion participant as a group administrator, they may assume that the status automatically bestows some level of authority on the group topic. Witness marks indicating formal and informal levels of knowledge would provide visual affirmation of authority type to support trustworthiness of posted content.

Research has noted the application of discourse markers in Twitter to promote identity (White, n.d.). A similar strategy in FB could provide verifiable 
credentials. A witness mark to indicate authority could be as simple as a visual enhancement to the username on the FB system. Consider the above example where the posting states "Vet here." Users would have a veterinary caduceus icon added to their posts within the FB group indicating they hold a health-related degree or official credentialing in the pet healthcare industry. Similar to the time witness mark, the icon-enhanced username would contribute to discerning trustworthiness of shared knowledge.

To this point proposed witness marks require FB system development for automatic visual representation. However, users can intentionally represent these issues as indicated through textual representation. Declarations of time and authority can be purposively incorporated into posts to promote cognitive authority. However, discoverability through search strategies remains elusive with this approach due to the SIR complications mentioned earlier.

With discussion of what is possible proposed above, I turn to existing system tools in FB that have the potential to promote discoverability and identification of cognitive authority if employed strategically by users. Application of tools such as \# and @ symbols serve to increase both cognitive authority and discoverability through identity promotion and content representation.

The hashtag (\#) is an intentional witness mark supported in the FB system. Hashtags were originally created for use in Internet Relay Chat (IRC) but were quickly engineered for use in Twitter. The intended purpose was to facilitate information retrieval in online social networks (OSN) by grouping information such as text content, images, and video through application of metadata to facilitate searches (Pandell, 2017). Through indexing, content becomes searchable. The hashtag was adopted by FB in 2013 in an attempt to increase discoverability of information. The evolution of the hashtag has resulted in expressions beyond meta-tagging for discoverability. Frequent observation reveals application of the hashtag to promote identity and perceptions of vanity irrelevant to search topics. For example, \#NFLfan labels a user as a fan of football while \#mycuteboy expresses a mother's emotion for a child. So the hashtag must be considered in the broader context of the post. These vanity expressions serve as witness marks however they fail to support cognitive authority and discoverability of secondhand knowledge.

Conceptualizing on the 'who' approach mentioned above, \#VetHere is a discursive approach that amalgamates presentation of authority and discoverability. Indexing of \#VetHere would label the user as having verifiable professional knowledge as well as when searched, it would reveal content of all posts thus labeled. The hashtag combined with identity promotes trustworthiness of information, be it authority or cognitive authority in nature. 
Consideration of time as a factor and its presentation via hashtag is less effective than identity promotion via the hashtag. Although presentable such as in "my dog was diagnosed with XX \#2weeks ago..." it will indicate limited time experience with the disease. And in turn "My rottie has been taking XXX [medication name] for a little over \#10years. Her appetite returned after a few weeks" identifies lengthy experience. Limitations on this approach include the fact that time is just one of many factors that contribute to cognitive authoritybuilding. Other factors that contribute to building cognitive authority include breadth, depth and intensity of experience as well as intentional knowledgebuilding activities such as research and reading.

A review of more than a thousand posts to the canine chronic disease FB group revealed that not one occurrence of a hashtag was found. However, highly effective in its original intent of knowledge sharing and discoverability, the hashtag as witness mark could be effectively augmented through a community informatics approach. Bonnici and $\mathrm{Ma}$ (in-press) propose a semi-automated tagging strategy for FB. Words commonly used in the community that are also frequent topics of inquiry could facilitate strategic tagging of posts. For example, in the canine chronic illness forum, an examination of over 1,000 randomly selected initiating posts reveals questions about medications used to treat the disease topic of the community. Thus the previously considered post about medication could be tagged as "My rottie has been taking \#medicationname for a little over 10 years. Her appetite returned after a few weeks." The hashtag would promote retrieval when the medication name is searched within the group site. The semi-automated tagging would be presented via a clickable hashtag (\#) button that presents a drop-down list of common community words with the name of the medicine being one choice. Ideally, introducing a user inter-tagging strategy where one user can assign hashtags to existing content posted by another would serve to increase aspects of cognitive authority and discoverability. Already used in some OSN such as Goodreads, Academia.edu, and Reddit, the strategy would be theoretically conceivable in FB. Consider the example post "My rottie has been taking XXX [medication name] for a little over \#10years. Her appetite returned after a few weeks." Another community member could add a hashtag promoting the medication information by tagging the post with \#medicationname rendering it searchable.

In sum, an intentional, expanded, and strategic approach to hashtag use in FB groups would serve to promote discovery of second-hand knowledge in turn decreasing anxiety and doubt in the user who is in an information-seeking crisis. Considering witness marks further, another existing FB symbol is also applicable.

Another discursive mark commonly applied in Twitter that informs a witness mark strategy is the @ symbol. Commonly used by Tweeters for phatic communion that lacks information exchange, the @ can also be used to prompt 
action by others (Miller, 2008). The @ is the only existing inter-tagging strategy in FB and thus it has the potential power to call upon cognitive authority. Tagging someone with@ to attract their attention to an inquiry that can be informed by their experience promotes secondhand knowledge dissemination. Employed to beg the attention of a specific user in the system, its application notifies the tagged user by posting to their FB space in the group. Examination of the same posts mentioned earlier reveals limited use of this intended approach. Only thee posts out of more than a thousand reviewed revealed use of the @ symbol. In each of the three cases, an initial inquiry had gone unaddressed for more than six hours. Replying to the originating inquiry post with @ tagging a specific user resulted in a posted contribution from the tagged individual within an hour's time for each of the three instances. Perhaps the frequency by which a FB group member is tagged could serve as an indicator of cognitive authority on the group topic or micro-discussions as delineated by hash-tagged topics.

\section{Conclusions}

Social information retrieval, like many other aspects of ubiquitously changing technologies, can be informed by consideration of user behaviors. In order to break the boundaries of thought, researchers must ponder the potential of systems beyond their current use. It is such an approach that gave birth to the various OSN considered and mentioned here.

A combination of conceptualized additions and existing FB labeling functions investigated through the lens of IxD could promote cognitive authority and discoverability. Icons to delineate authority roles and time experience informed by IxD as well as existing FB functions such as hashtag (\#) and @ are offered as potential witness marks. The proposed strategies would serve to ameliorate successful information-seeking efforts, particularly in high-stress, lifethreatening conditions. However, these ideas of what could be are offered for consideration not only in the milieu of FB groups, but also for FB in general as well as other OSN. Strategies that promote cognitive authority and discoverability serve to cull the s- from a community of secondhand knowledge.

\section{References}

Bonnici, L.J. \& Ma, J. (in-press). Social Information Discoverability in Facebook Groups: The Need for Linked Data Strategies. Manuscript submitted for publication. 
Card, S. K., Moran, T.P., \& Newell, A. (1980). The keystroke-level model for user performance time with interactive systems. Communications of the ACM. 23(7), 396-410.

Cooper, A., Reiman, R., \& Cronin, D. (2007). About Face 3: The Essentials of Interaction Design. Indianapolis, Indiana: Wiley.

Maltese, J. (2017). Fixing someone else's work: AV Witness Marks. Sound \& Communications, Retrieved from http://www.soundandcommunications.com/fixing-someone-elses-work-avwitness-marks/

Miller, V. (2008). New Media, Networking and Phatic Culture. Convergence: The International Journal of Research into New Media Technologies, 14(4), 387-400.

Pandell, L. (2017). An oral history of the \#hashtag. Wired Magazine, Retrieved from https://www.wired.com/2017/05/oral-history-hashtag/

Rogers, Y., Sharp, H., \& Preece, J. (2011). Interaction Design: Beyond humancomputer interaction ( $3^{\text {rd }}$ ed.). West Sussex, U.K.: John Wiley \& Sons Ltd.

White, J. (n.d.). Membership categorization and identity work achieved by discourse markers on Twitter [Blog post]. Retrieved from https://mediamum.wordpress.com/research-papers andpresentations/identity-work-performed-on-twitter-using-hashtags-andretweets/ 